\title{
Imaging in abusive head trauma: an in-depth look at current issues
}

\author{
Catherine Adamsbaum • Marilyn Barr
}

Received: 28 April 2014 / Accepted: 21 May 2014

(C) Springer-Verlag Berlin Heidelberg 2014

We are honored to introduce this special issue on abusive head trauma. First, we would like to warmly thank the authors and their collaborators who have kindly shared their excellent work. All of the articles have been written by brilliant authors who are recognized experts in this field.

The authors are not only radiologists but also pediatricians, pathologists, neurosurgeons, epidemiologists, forensic doctors and pathologists. This melting pot of expertise exquisitely illustrates the need for close communication if we are to move forward in our understanding of abusive head trauma. The International Conference on Abusive Head Trauma, organized by the National Center for Shaken Baby Syndrome (NCSBS) in partnership with the European Organizing Committee, highlights the importance of sharing information and new insights on this critical topic.

Child abuse, specifically head trauma, continues to be of special concern worldwide. Recent court decisions in the United States, Canada and the United Kingdom have demonstrated some confusion about the evidence presented in cases of alleged abusive head trauma. However, medical professionals in all these countries agree that although abusive head trauma cases can be complex, there is no doubt that such abuse is a tragic reality.

Pediatric radiologists often play a key role in the early diagnosis of suspected inflicted injury by identifying subdural

\section{Adamsbaum $(\square)$}

European Organizing Committee, Hôpital Bicêtre APHP, Faculté de Médecine Paris Sud, 78 rue du Général Leclerc,

Le Kremlin Bicêtre 94275, Cedex, France

e-mail: adamsbaum.catherine@gmail.com

\section{Barr}

National Center on Shaken Baby Syndrome, 1433 North 1075

West Suite 110, Farmington, UT 84025, USA hematomas, parenchymal injuries and fractures whose patterns have been exhaustively described in textbooks and numerous reports. The imaging articles included in this issue focus on specific points regarding spinal injuries, skeletal injuries, relative value of CT and MRI in the scope of abusive head trauma evaluation, and the limits in dating such injuries.

This major involvement of pediatric radiologists is achieved through the commitment of the European Society of Paediatric Radiology (ESPR) (child abuse taskforce) and Pediatric Radiology (the official journal of the ESPR, the Society for Pediatric Radiology, the Asian and Oceanic Society for Paediatric Radiology and the Latin American Society of Pediatric Radiology). This journal has recently created an editorial board group on child abuse.

In this effort, it is very important to improve our understanding of how abusive head trauma occurs and to gain insights of what is needed to come to an accurate diagnosis as early as possible. For example, understanding the reasons a caretaker or parent might shake a child could be invaluable to protect the child and to help in establishing effective prevention initiatives. The pattern of crying known as the "normal infant crying curve" is nicely described by Dr. Ronald G. Barr elsewhere in this issue, and awareness would benefit pediatric radiologists.

Keeping in mind that differential diagnoses are of the utmost importance to avoid tragic mistakes, this issue offers views from various outstanding specialists, pediatricians and radiologists who contribute to that topic.

Expert pathologists have shared their knowledge of macroscopic data in abusive head trauma. This information is very helpful when looking for subtle abnormalities such as bridging vein thrombosis when differentiating accidental from inflicted head trauma.

This issue includes key points for pediatric radiologists to consider such as findings in retinal hemorrhages, long-term outcomes, injury mechanisms, sports-related injuries, risk 
factors, international issues on inflicted injury and the lessons learned in public hearings in France.

We hope this issue will also be of interest to pediatricians and other sub-specialists, medical students and residents, as well as judges, prosecutors and social workers, all of whom work with abused children.

Above all, our hope is to help to prevent abusive head trauma and its devastating consequences.
Acknowledgments We would like to warmly thank the European Organizing Committee: Anne Laurent-Vannier, MD, and Caroline ReySalmon, MD, for their hard work and support in the preparation of the conference and of this issue. Additionally, we appreciate Danielle Vazquez for her invaluable help in the preparation of the European Conference on Abusive Head Trauma and Pascale Zerbini for the preparation of the manuscripts of this issue.

Conflicts of interest None 